\title{
TWO YEARS CLINICAL EVALUATION OF A BULK FILL COMPOSITE RESIN COMPARED WITH A NANOHYBRIDE INCREMENTAL FILL COMPOSITE RESIN IN CLASS II CAVITIES
}

\author{
Reham M. Attia*
}

\begin{abstract}
Abstract: The objective of this study was to determine the two years clinical performance of a bulk fill composite and conventional nanohybride incremental fill composite in class II cavities.

Materials and methods: A total of 60 class $\Pi$ cavities were restored with either bulk fill material (Tetric EvoCeram bulk fill) and self-etch adhesive (AdheSE adhesive) or an incremental resin composite (Tetric N-Ceram Nano-hybrid) and self-etch adhesive (AdheSE adhesive). In group I: The restorative material Tetric EvoCeram Bulk-Fill was applied in one bulk. In group П: Tetric $\mathrm{N}$-Ceram Nano-hybrid was applied using an incremental filling technique starting at the gingival wall. All restorations were clinically evaluated at periods of 1 week (initial recall), after six months, after one year, after eighteen months and at the end of two years. Assessment was done according to the modified United States Public Health Service (USPHS) criteria. Data were statistically analyzed using Friedman test and Fisher's exact at $\mathrm{P}<0.05$ level of significance.
\end{abstract}

Results: There was no significant difference between group I and group $\Pi$ regarding all tested criteria at different follow up periods; where $\mathrm{P}>0.05$.

Conclusion: Bulk fill composite (Tetric EvoCeram bulk fill) and incremental fill composite resin (Tetric N-Ceram Nano-hybrid) exhibited comparable acceptable clinical performance after two years of evaluation.

KEY WORDS: Bulk fill composite, Clinical evaluation, Incremental fill.

\section{INTRODUCTION}

Resin composites are currently viewed as the material of choice for restoring posterior teeth even in stress bearing areas using the minimally invasive cavity preparation design ${ }^{[1]}$. It is one of the most widely used modern dental restorative materials. Resin composite has many advantages such as outstanding esthetics and easiness of handling ${ }^{[2]}$. Their properties have been enhanced progressively to augment their stability in the oral environment which is characterized by different challenges ${ }^{[3]}$.

\footnotetext{
* Lecturer of Operative Dentistry, Conservative Dentistry Department, Faculty of Dentistry, Zagazig University.
} 
The polymerization of resin-based composites leads to stresses due to their contraction during the polymerization process. The stress occurs when the resin composite material is rigid and resist adequate plastic flow to compensate for the original volume ${ }^{[1]}$.

The degree of polymerization contraction stresses is extremely dependent on the viscoelastic properties of the resin based composite material. In clinical situations, these stresses possibly affect marginal quality ${ }^{[4]}$. When marginal quality is adversely affected many drawbacks such as leakage, recurrent caries and irritation of the pulp may take place. Marginal integrity is very important and should be the main intention for operator ${ }^{[5]}$.

The incremental placement technique of composite resin is the most frequently used method clinically to keep away from depth-of-cure limits and to conquer the stress ${ }^{[6]}$. On the other hand, in addition to these advantages, there are a number of disadvantages coupled with the use of an incremental approach to placing resins in the cavity, for example, voids can be trapped between the increments ${ }^{[2,7,8]}$.

A recent invention of bulk-fill resin composites has been introduced. There are two main categories of bulk-fill composites, one indicated for use as posterior restorations in one bulk, the other bulk fill materials are applied under appropriate posterior composites as underlining or base materials ${ }^{[9]}$. The bulk-fill composites present reduced polymerization contraction stresses ${ }^{[10]}$. If bulk fill composite resin is applied in one bulk and then cured, the procedure will decrease the time of restoration, as well as air voids trap is markedly diminished. The resultant final restoration will exhibit a good quality ${ }^{[11]}$.

Different methods are used to achieve maximum depth of cure and decrease the polymerization shrinkage stress ${ }^{[6]}$. The monomer characterized by stress relieving properties, novel photoinitiators and prepolymerized particles leads to marked reduction of polymerization shrinkage ${ }^{[12]}$. Better light transmission and adequate depth of cure may be achieved by increased translucency of the resin ${ }^{[1]}$. Many in vitro studies were carried out and compared the bulk fill composite versus the incremental fill one ${ }^{[9-12]}$. However, the clinical oral conditions presented many challenges which cannot simulate perfectly in invitro studies ${ }^{[17]}$.

Despite the bulk fill composite resin materials show many advantages, but the incremental fill composite is widely used clinically. So, the objective of this clinical study was to evaluate the clinical performance of a bulk fill resin composite and compare it with a conventional incremental fill nanohybride resin composite for two years.

\section{MATERIALS AND METHODS}

30 patients, aged between 30 - 40 years old (13 female and 17 male), were selected to participate in this study according to detailed exclusion and inclusion criteria. Nature, objectives and procedures of the study were explained to the patients. Written informed consent was obtained from all patients before their participation in the clinical evaluation. Inclusion criteria were: (1) patients who could be present for further periodic follow-up were selected.

(2) Presence of permanent molars requiring treatment of class II primary carious lesions. (3) All the selected teeth in the study were in occlusion with natural dentition and had proximal contact with neighbouring teeth. (4) Good oral hygiene. (5) Possibility for application of rubber dam during restoration.

Exclusion criteria were: (1) Poor oral hygiene. (2) Heavy bruxism habit or presence of any parafunction habits. (3) Periodontal problems. (4) Any allergic reactions against any components of the materials used in the study. (5) Pathologic pulpal involvement manifested as induced or spontaneous pain or the presence of non vital tooth. (6) Fractured or evidently cracked teeth. (7) Defective restorations neighbouring or opposite the tooth 
selected for the current research. (8) Patients with high caries index or high plaque index. (9) Atypical extrinsic staining of teeth. (10) Teeth with defects or lesions requiring other operative interventions. (11) History of severe medical complications such as xerostomia. (12) Pregnant or lactating women ${ }^{[13-15]}$. Patients were given oral hygiene instructions. Each patient received at least one pair of restoration representing the two tested materials. A total of 60 class $\Pi$ cavities were restored with either bulk fill material (Tetric EvoCeram bulk fill) and self-etch adhesive (AdheSE adhesive) or an incremental resin composite (Tetric N-Ceram Nano-hybrid) and selfetch adhesive (AdheSE adhesive). The materials used in the study are shown in table (1).

The distribution of materials and tooth locations were randomized to equally distribute materials into some important variables such as tooth type and position, in such a way that minimized the effects of those factors ${ }^{[16]}$.
Restorative procedures were performed under local anaesthesia if necessary. The teeth were completely isolated using rubber dam (Dental rubber dam, pure Latex. Health Co International, Inc., Boston, U.S.A). Class II cavities were prepared where the cavity design was limited to eradicate carious tissues from primary carious lesions ${ }^{[17]}$. Adhesive cavity design was performed where the inner angles of the cavities were rounded and the margins were not beveled. The carious tissues were removed using diamond burs of the suitable size at high speed (Medin,a.s., Valachovicka619, Czech Republic) under profuse water cooling system. A thin layer of calcium hydroxide liner (Dycal, Dentsply/Caulk, Milford, DE, USA) was placed in the deepest part of the cavity to protect the pulpal tissue.

After the cavity was prepared and the shade of resin was selected, all restorations were performed using a pre-curved metallic sectional matrix (Palodent Sectional Matrix System Dentsply/Caulk, Milford, DE, USA), associated with a separating ring

TABLE (1): Materials: composition, manufacturers, and website of tested materials

\begin{tabular}{|c|c|c|c|}
\hline Material & Chemical composition & Manufacturer & Website \\
\hline $\begin{array}{l}\text { Tetric } \\
\text { EvoCeram } ® \\
\text { Bulk Fill } \\
\text { composite }\end{array}$ & $\begin{array}{l}\text { The monomer matrix is composed of dimethacrylates }(20-21 \% \\
\text { weight). Thefillers contain barium glass, ytterbium trifluoride, } \\
\text { mixed oxide and prepolymer ( } 79-81 \% \text { weight). Additional contents: } \\
\text { additives, catalysts, stabilizers and Pigments }(<1.0 \% \text { weight). The } \\
\text { total content of inorganic fillers is } 76-77 \% \text { Weight or } 53-54 \% \\
\text { volume. The particle size of the inorganic fillers is between } 40 \mathrm{~nm} \\
\text { and } 3,000 \mathrm{~nm} \text { with a mean particle size of } 550 \mathrm{~nm} \text {. }\end{array}$ & $\begin{array}{l}\text { IvoclarVivadent, } \\
\text { Schaan, } \\
\text { Liechtenstein }\end{array}$ & $\begin{array}{l}\text { www.ivoclarvivadent. } \\
\text { com }\end{array}$ \\
\hline $\begin{array}{l}\text { Tetric } \AA \\
\text { N-Ceram } \\
\text { Nano-hybrid } \\
\text { incremental } \\
\text { composite }\end{array}$ & $\begin{array}{l}\text { Dimethacrylates }(19-20 \mathrm{wt} \%) \text {. The fillers contain barium glass, } \\
\text { ytterbium trifluoride, mixed oxide and Copolymers }(80-81 \mathrm{wt} . \%) \text {. } \\
\text { Additives, catalysts, stabilizers and pigments are additionalcontents } \\
(<1 \mathrm{wt} \%) \text {. The total content of inorganic fillers is } 55-57 \mathrm{vol} \% \text {. The } \\
\text { particle size of inorganic fillers is between } \\
40 \mathrm{~nm} \text { and } 3000 \mathrm{~nm} \text {. }\end{array}$ & $\begin{array}{l}\text { IvoclarVivadent, } \\
\text { Schaan, } \\
\text { Liechtenstein }\end{array}$ & $\begin{array}{l}\text { www.ivoclarvivadent. } \\
\text { com }\end{array}$ \\
\hline $\begin{array}{l}\text { AdheSE } \\
\text { adhesive }\end{array}$ & $\begin{array}{l}\text { Primer: Phosphonic acid acrylate Bis-acrylamide. Water Initiators } \\
\text { and stabilizers. } \\
\text { Bond: Dimethacrylates Hydroxyethyl methacrylate, Highly } \\
\text { dispersed silicon dioxide, Initiators and stabilizers, Activator } \\
\text { Solvent Initiators. }\end{array}$ & $\begin{array}{l}\text { IvoclarVivadent, } \\
\text { Schaan, } \\
\text { Liechtenstein }\end{array}$ & $\begin{array}{l}\text { www.ivoclarvivadent. } \\
\text { com }\end{array}$ \\
\hline
\end{tabular}


and a wooden wedge to establish the anatomical shape and proximal contacts of the teeth. A total of 60 restorations were placed in the cavities of the 30 patients. The participants were not aware of which type of composite restoration was used in which cavity. Restorations were performed according to the manufacturer's instructions as follows: Group (I): AdheSE primer was applied to the prepared cavity for 30 seconds, then dispersed with a stream of air. Uniform thin layer of the adhesive was applied on all the prepared surface and light cured for 10 seconds (cromalux ${ }^{100}$ curing unit. MEGA-PHYSIK DENTAL. Rastatt. Germany). The output of the curing unit was checked after each patient with a curing radiometer (Optilux radiometer Kerr-USA). The restorative material Tetric EvoCeram Bulk-Fill was applied in one bulk and cured for 20s.

Group (П): AdheSE primer was applied to the prepared cavity as in group I. Tetric N-Ceram Nano-hybrid was applied using an incremental filling technique starting at the gingival wall. Each increment was polymerized for 20 seconds.

For all restoration, occlusal adjustments were made using articulating paper. Finishing was accomplished by diamond finishing bur (T\&F hybrid points kit, diamond points. Shofu inc., Kyoto. Japan) then polished using rubber points (Kenda dental polishers, Liechtenstein). Final polishing was done using ultra polishing SuperSnap kit (Super -Snap, super buff set Shofu inc., Kyoto. Japan). Distribution of the teeth restored in the study is summarized in table (2).

Table (2): Distribution of the teeth restored in the study:

\begin{tabular}{|c|c|c|c|c|}
\hline Group & $\begin{array}{c}\text { Upper } \\
\text { premolar }\end{array}$ & $\begin{array}{c}\text { Upper } \\
\text { molar }\end{array}$ & $\begin{array}{c}\text { Lower } \\
\text { premolar }\end{array}$ & $\begin{array}{c}\text { Lower } \\
\text { molar }\end{array}$ \\
\hline Group I & 8 & 4 & 5 & 13 \\
\hline Group II & 12 & 5 & 3 & 10 \\
\hline
\end{tabular}

All restorations were clinically evaluated at periods of 1 week (initial recall), after six months, after one year, after 18 months and at 2 years. Assessment was done according to the modified United States Public Health Service (USPHS) criteria table (3) ${ }^{[18-20]}$. Assessment was carried out by two independent evaluators. Each of these evaluators was not the same individual who placed the restorations and was unaware of the materials used in this double-blind study. If there were any discrepancies between the two examiners, they should evaluate the restorations together and determine the score by consensus ${ }^{[21,22]}$. The recorded data obtained for each tested criterion at each follow up period were collected and statistically analyzed using SPSS (Statistical Package for the Social Sciences) soft ware version 20.0. Friedman test and Fisher's exact were used to compare the changes across different time points and between the two tested groups at different follow up periods. The level of significance was $(\mathrm{P}<0.05)$.

TABLE (3): Modified USPHS criteria:

\begin{tabular}{|c|c|c|}
\hline 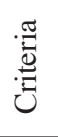 & $\begin{array}{l}\text { Analogous } \\
\text { U S P H S } \\
\text { criteria }\end{array}$ & Description \\
\hline \multirow{4}{*}{ 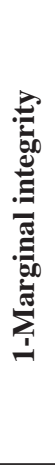 } & $\begin{array}{l}\text { - Alpha } \\
\text { (A) }\end{array}$ & $\begin{array}{l}\text { - No visible crevice or so small that } \\
\text { the probe just catch it and not fall } \\
\text { in. }\end{array}$ \\
\hline & $\begin{array}{l}\text { - Bravo } \\
\text { (B) }\end{array}$ & $\begin{array}{l}\text { - Explorer tip fall into crevice but the } \\
\text { dentin was not exposed. }\end{array}$ \\
\hline & $\begin{array}{l}\text { - Charlie } \\
\text { (C) }\end{array}$ & $\begin{array}{l}\text { - Explorer penetrates into a crevice } \\
\text { that is of depth that exposes dentin } \\
\text { or base. }\end{array}$ \\
\hline & $\begin{array}{l}\text {-Delta } \\
\text { (D) }\end{array}$ & - Immediate replacement necessary \\
\hline \multirow{4}{*}{ 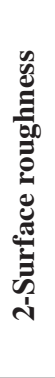 } & $\begin{array}{l}\text { - Alpha } \\
\text { (A) }\end{array}$ & $\begin{array}{l}\text { - Surface is smooth as the surrounding } \\
\text { enamel. }\end{array}$ \\
\hline & $\begin{array}{l}\text { - Bravo } \\
\text { (B) }\end{array}$ & $\begin{array}{l}\text { - Surface is rougher than surrounding } \\
\text { enamel. }\end{array}$ \\
\hline & $\begin{array}{l}\text { - Charlie } \\
\text { (C) }\end{array}$ & - Surface is very rough. \\
\hline & $\begin{array}{l}\text {-Delta } \\
\text { (D) }\end{array}$ & - Immediate replacement necessary \\
\hline
\end{tabular}




\begin{tabular}{|c|c|c|}
\hline \multirow{4}{*}{ 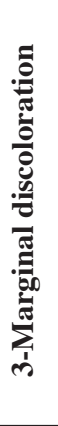 } & $\begin{array}{l}\text { - Alpha } \\
\text { (A) }\end{array}$ & $\begin{array}{l}\text { - No discoloration anywhere on the } \\
\text { margin between the restoration and } \\
\text { the tooth structure. }\end{array}$ \\
\hline & $\begin{array}{l}\text { - Bravo } \\
\text { (B) }\end{array}$ & $\begin{array}{l}\text { - Presence of marginal discoloration, } \\
\text { limited and not extended }\end{array}$ \\
\hline & $\begin{array}{l}\text { - Charlie } \\
\text { (C) }\end{array}$ & $\begin{array}{l}\text { - Evident marginal discoloration, } \\
\text { penetrated toward the pulp chamber. }\end{array}$ \\
\hline & $\begin{array}{l}\text {-Delta } \\
\text { (D) }\end{array}$ & - Immediate replacement necessary. \\
\hline \multirow{3}{*}{ 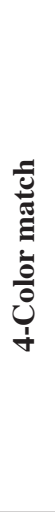 } & $\begin{array}{l}\text { - Alpha } \\
\text { (A) }\end{array}$ & $\begin{array}{l}\text { - The restoration matches the } \\
\text { adjacent tooth structure in color, } \\
\text { shade or translucency }\end{array}$ \\
\hline & $\begin{array}{l}\text { - Bravo } \\
\text { (B) }\end{array}$ & $\begin{array}{l}\text { - Mismatch in color, shade or } \\
\text { translucency between the } \\
\text { restoration and the adjacent tooth } \\
\text { within an acceptable range }\end{array}$ \\
\hline & $\begin{array}{l}\text { - Charlie } \\
\text { (C) }\end{array}$ & $\begin{array}{l}\text { - The mismatch in color and } \\
\text { translucency is outside the } \\
\text { acceptable range of tooth color and } \\
\text { translucency. }\end{array}$ \\
\hline \multirow{4}{*}{ 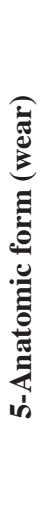 } & $\begin{array}{l}- \text { Alpha } \\
\text { (A) }\end{array}$ & $\begin{array}{l}\text { - Restoration is continuous with } \\
\text { existing anatomic form. }\end{array}$ \\
\hline & $\begin{array}{l}\text { - Bravo } \\
\text { (B) }\end{array}$ & $\begin{array}{l}\text { - Restoration is discontinuous with } \\
\text { existing anatomic form, but missing } \\
\text { material is not sufficient to expose } \\
\text { dentin or base. }\end{array}$ \\
\hline & $\begin{array}{l}\text { - Charlie } \\
\text { (C) }\end{array}$ & $\begin{array}{l}\text { Sufficient restorative } \\
\text { material is missing to expose dentin } \\
\text { or base }\end{array}$ \\
\hline & $\begin{array}{l}\text {-Delta } \\
\text { (D) }\end{array}$ & - Immediate replacement necessary. \\
\hline
\end{tabular}

\begin{tabular}{|c|c|c|}
\hline \multirow{2}{*}{ 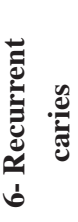 } & $\begin{array}{l}\text { - Alpha } \\
\text { (A) }\end{array}$ & $\begin{array}{l}\text { - No evidence for caries contiguous } \\
\text { with the restoration margins. }\end{array}$ \\
\hline & $\begin{array}{l}\text { - Bravo } \\
\text { (B) }\end{array}$ & $\begin{array}{l}\text { - Caries contiguous with the } \\
\text { restoration margin. }\end{array}$ \\
\hline \multirow{4}{*}{ 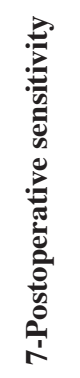 } & $\begin{array}{l}\text { - Alpha } \\
\text { (A) }\end{array}$ & - Not present. \\
\hline & $\begin{array}{l}\text { - Bravo } \\
\text { (B) }\end{array}$ & $\begin{array}{l}\text { - Sensitive but diminishing in } \\
\text { intensity. }\end{array}$ \\
\hline & $\begin{array}{l}\text { - Charlie } \\
\text { (C) }\end{array}$ & $\begin{array}{l}\text { - Constant sensitivity, not diminishing } \\
\text { in intensity. }\end{array}$ \\
\hline & $\begin{array}{l}\text { - Delta } \\
\text { (D) }\end{array}$ & - Immediate replacement necessary. \\
\hline
\end{tabular}

\section{RESULTS:}

Regarding the marginal integrity and marginal discoloration of the restoration; Alpha score decreased in both groups throughout the follow up periods. There was no significant difference in group I and in group II during the period of study. A comparison between the two groups at different follow up periods revealed no statistically significant difference at different follow up periods where $\mathrm{P}$ value was $1.00,0.67,0.84$ and 0.63 at six month $\mathrm{s}, 12$ months, 18 months and finally at 24 months. At base line; Alpha score was recorded in all the restoration of both two tested groups; so the difference between the two groups was not computed. 
TABLE (4): Results of clinical evaluation regarding marginal integrity and marginal discoloration of restoration

\begin{tabular}{|c|c|c|c|c|c|c|c|c|c|c|c|c|}
\hline \multirow{2}{*}{ Scores } & \multicolumn{2}{|c|}{$\begin{array}{l}\text { Baseline } \\
\text { No. (30) }\end{array}$} & \multicolumn{2}{|c|}{$\begin{array}{l}6 \text { months } \\
\text { No. (30) }\end{array}$} & \multicolumn{2}{|c|}{$\begin{array}{l}12 \text { months } \\
\text { No. (30) }\end{array}$} & \multicolumn{2}{|c|}{$\begin{array}{l}18 \text { months } \\
\text { No. (30) }\end{array}$} & \multicolumn{2}{|c|}{$\begin{array}{l}24 \text { months } \\
\text { No. (30) }\end{array}$} & \multirow{2}{*}{$\begin{array}{c}\text { Friedman } \\
\text { test }\end{array}$} & \multirow[t]{2}{*}{ P-value } \\
\hline & No & $\%$ & No & $\%$ & No & $\%$ & No & $\%$ & No & $\%$ & & \\
\hline \multicolumn{13}{|l|}{ Group I } \\
\hline Alpha & 30 & 100.0 & 28 & 93.3 & 28 & 93.3 & 25 & 83.3 & 25 & 83.3 & \multirow{4}{*}{10.54} & \multirow{4}{*}{0.23} \\
\hline Bravo & 0 & 0.0 & 2 & 6.7 & 2 & 6.7 & 5 & 16.7 & 4 & 13.3 & & \\
\hline Charlie & 0 & 0.0 & 0 & 0.0 & 0 & 0.0 & 0 & 0.0 & 1 & 3.3 & & \\
\hline Delta & 0 & 0.0 & 0 & 0.0 & 0 & 0.0 & 0 & 0.0 & 0 & 0.0 & & \\
\hline \multicolumn{13}{|l|}{ Group II } \\
\hline Alpha & 30 & 100.0 & 27 & 90.0 & 26 & 86.7 & 24 & 80.0 & 22 & 73.3 & \multirow{4}{*}{15.95} & \multirow{4}{*}{0.15} \\
\hline Bravo & 0 & 0.0 & 3 & 10.0 & 4 & 13.3 & 6 & 20.0 & 6 & 20.0 & & \\
\hline Charlie & 0 & 0.0 & 0 & 0.0 & 0 & 0.0 & 0 & 0.0 & 0 & 0.0 & & \\
\hline Delta & 0 & 0.0 & 0 & 0.0 & 0 & 0.0 & 0 & 0.0 & 0 & 0.0 & & \\
\hline Fisher's exact & \multicolumn{2}{|c|}{-} & \multicolumn{2}{|c|}{0.00} & \multicolumn{2}{|c|}{0.19} & \multicolumn{2}{|c|}{0.35} & \multicolumn{2}{|c|}{0.93} & & \\
\hline P-value & \multicolumn{2}{|c|}{ - } & \multicolumn{2}{|c|}{1.00} & \multicolumn{2}{|c|}{0.67} & \multicolumn{2}{|c|}{0.84} & \multicolumn{2}{|c|}{0.63} & & \\
\hline
\end{tabular}

TABLE (5): Results of clinical evaluation regarding surface roughness of restoration

\begin{tabular}{|c|c|c|c|c|c|c|c|c|c|c|c|c|}
\hline \multirow[t]{2}{*}{$\begin{array}{r}\text { Follow up } \\
\text { period }\end{array}$} & \multicolumn{2}{|c|}{$\begin{array}{l}\text { Baseline } \\
\text { No. (30) }\end{array}$} & \multicolumn{2}{|c|}{$\begin{array}{l}6 \text { months } \\
\text { No. }(30)\end{array}$} & \multicolumn{2}{|c|}{$\begin{array}{l}12 \text { months } \\
\text { No. (30) }\end{array}$} & \multicolumn{2}{|c|}{$\begin{array}{l}18 \text { months } \\
\text { No. }(30)\end{array}$} & \multicolumn{2}{|c|}{$\begin{array}{c}24 \text { months } \\
\text { No. }(30)\end{array}$} & \multirow[t]{2}{*}{$\begin{array}{c}\text { Friedman } \\
\text { test }\end{array}$} & \multirow[t]{2}{*}{ P-value } \\
\hline & No & $\%$ & No & $\%$ & No & $\%$ & No & $\%$ & No & $\%$ & & \\
\hline \multicolumn{13}{|l|}{ Group I } \\
\hline Alpha & 30 & 100.0 & 28 & 93.3 & 28 & 93.3 & 25 & 83.3 & 25 & 83.3 & \multirow{4}{*}{10.54} & \multirow{4}{*}{0.23} \\
\hline Bravo & 0 & 0.0 & 2 & 6.7 & 2 & 6.7 & 5 & 16.7 & 4 & 13.3 & & \\
\hline Charlie & 0 & 0.0 & 0 & 0.0 & 0 & 0.0 & 0 & 0.0 & 1 & 3.3 & & \\
\hline Delta & 0 & 0.0 & 0 & 0.0 & 0 & 0.0 & 0 & 0.0 & 0 & 0.0 & & \\
\hline \multicolumn{13}{|l|}{ Group II } \\
\hline Alpha & 30 & 100.0 & 27 & 90.0 & 25 & 83.3 & 27 & 80.0 & 26 & 80.0 & \multirow{4}{*}{17.31} & \multirow{4}{*}{0.14} \\
\hline Bravo & 0 & 0.0 & 3 & 10.0 & 5 & 16.7 & 2 & 6.7 & 2 & 6.7 & & \\
\hline Charlie & 0 & 0.0 & 0 & 0.0 & 0 & 0.0 & 1 & 3.3 & 2 & 6.7 & & \\
\hline Delta & 0 & 0.0 & 0 & 0.0 & 0 & 0.0 & 0 & 0.0 & 0.0 & 0.0 & & \\
\hline $\begin{array}{c}\text { Fisher's } \\
\text { exact }\end{array}$ & \multicolumn{2}{|c|}{-} & \multicolumn{2}{|c|}{1.02} & \multicolumn{2}{|c|}{0.65} & \multicolumn{2}{|c|}{3.52} & \multicolumn{2}{|c|}{2.19} & & \\
\hline P-value & \multicolumn{2}{|c|}{ - } & \multicolumn{2}{|c|}{0.60} & \multicolumn{2}{|c|}{0.42} & \multicolumn{2}{|c|}{0.17} & \multicolumn{2}{|c|}{0.53} & & \\
\hline
\end{tabular}

Table (6) revealed the scores of restorations of color match. There was no statistically significant difference between the two tested groups at the four recall period. The difference among the different recall periods of each tested group was not statistically significant as calculated by Friedman test in group I and group $\Pi$ where $P$ value was equal to 0.40 and 0.32 respectively. 
TABLE (6): Results of clinical evaluation regarding color match of restorations.

\begin{tabular}{|c|c|c|c|c|c|c|c|c|c|c|c|c|}
\hline \multirow{2}{*}{\begin{tabular}{|r} 
Follow up \\
period
\end{tabular}} & \multicolumn{2}{|c|}{$\begin{array}{l}\text { Baseline } \\
\text { No. (30) }\end{array}$} & \multicolumn{2}{|c|}{$\begin{array}{l}6 \text { months } \\
\text { No. }(30)\end{array}$} & \multicolumn{2}{|c|}{$\begin{array}{l}12 \text { months } \\
\text { No. (30) }\end{array}$} & \multicolumn{2}{|c|}{$\begin{array}{l}18 \text { months } \\
\text { No. (30) }\end{array}$} & \multicolumn{2}{|c|}{$\begin{array}{l}24 \text { months } \\
\text { No. (30) }\end{array}$} & \multirow{2}{*}{$\begin{array}{c}\text { Friedman } \\
\text { test }\end{array}$} & \multirow[t]{2}{*}{ P-value } \\
\hline & No & $\%$ & No & $\%$ & No & $\%$ & No & $\%$ & No & $\%$ & & \\
\hline \multicolumn{13}{|l|}{ Group I } \\
\hline Alpha & 29 & 96.7 & 28 & 93.3 & 28 & 93.3 & 25 & 83.3 & 25 & 83.3 & \multirow{4}{*}{8.38} & \multirow{4}{*}{0.40} \\
\hline Bravo & 1 & 3.3 & 2 & 6.7 & 2 & 6.7 & 5 & 16.7 & 4 & 13.3 & & \\
\hline Charlie & 0 & 0.0 & 0 & 0.0 & 0 & 0.0 & 0 & 0.0 & 1 & 3.3 & & \\
\hline Delta & 0 & 0.0 & 0 & 0.0 & 0 & 0.0 & 0 & 0.0 & 0 & 0.0 & & \\
\hline \multicolumn{13}{|l|}{ Group II } \\
\hline Alpha & 30 & 100.0 & 27 & 90.0 & 26 & 86.7 & 24 & 80.0 & 22 & 73.3 & \multirow{4}{*}{13.76} & \multirow{4}{*}{0.32} \\
\hline Bravo & 0 & 0.0 & 3 & 10.0 & 3 & 10.0 & 3 & 10.0 & 3 & 10.0 & & \\
\hline Charlie & 0 & 0.0 & 0 & 0.0 & 1 & 3.3 & 3 & 10.0 & 3 & 10.0 & & \\
\hline Delta & 0 & 0.0 & 0 & 0.0 & 0 & 0.0 & 0 & 0.0 & 0.0 & 0.0 & & \\
\hline $\begin{array}{c}\text { Fisher's } \\
\text { exact }\end{array}$ & \multicolumn{2}{|c|}{0.00} & \multicolumn{2}{|c|}{1.02} & \multicolumn{2}{|c|}{1.27} & \multicolumn{2}{|c|}{3.52} & \multicolumn{2}{|c|}{2.19} & & \\
\hline P-value & \multicolumn{2}{|c|}{1.00} & \multicolumn{2}{|c|}{0.60} & \multicolumn{2}{|c|}{0.53} & \multicolumn{2}{|c|}{0.17} & \multicolumn{2}{|c|}{0.53} & & \\
\hline
\end{tabular}

In spite that there was loss of anatomic form of restoration in both groups as shown in table (7), there was no statistically significant difference between the two groups at each recall period. Friedman test showed that the difference was not statistically significant among the different recall periods in both tested groups where $\mathrm{p}=0.23$ and 0.16 regarding group I and group II respectively .

TABLE (7): Results of clinical evaluation regarding anatomic form of restorations.

\begin{tabular}{|c|c|c|c|c|c|c|c|c|c|c|c|c|}
\hline \multirow{2}{*}{\begin{tabular}{|c} 
Follow up \\
period \\
Scores \\
\end{tabular}} & \multicolumn{2}{|c|}{$\begin{array}{l}\text { Baseline } \\
\text { No. (30) }\end{array}$} & \multicolumn{2}{|c|}{$\begin{array}{l}6 \text { months } \\
\text { No. (30) }\end{array}$} & \multicolumn{2}{|c|}{$\begin{array}{l}12 \text { months } \\
\text { No. (30) }\end{array}$} & \multicolumn{2}{|c|}{$\begin{array}{l}18 \text { months } \\
\text { No. (30) }\end{array}$} & \multicolumn{2}{|c|}{$\begin{array}{l}24 \text { months } \\
\text { No. (30) }\end{array}$} & \multirow[t]{2}{*}{$\begin{array}{c}\text { Friedman } \\
\text { test }\end{array}$} & \multirow[t]{2}{*}{ P-value } \\
\hline & No & $\%$ & No & $\%$ & No & $\%$ & No & $\%$ & No & $\%$ & & \\
\hline Group I & \multirow[b]{2}{*}{30} & \multirow[b]{2}{*}{100.0} & \multirow[b]{2}{*}{28} & \multirow[b]{2}{*}{93.3} & \multirow[b]{2}{*}{25} & \multirow[b]{2}{*}{83.3} & \multirow[b]{2}{*}{25} & \multirow[b]{2}{*}{83.3} & \multirow[b]{2}{*}{25} & \multirow[b]{2}{*}{76.7} & & \\
\hline Alpha & & & & & & & & & & & \multirow{4}{*}{10.52} & \multirow{4}{*}{0.23} \\
\hline Bravo & 0 & 0.0 & 2 & 6.7 & 5 & 16.3 & 5 & 16.3 & 5 & 16.3 & & \\
\hline Charlie & 0 & 0.0 & 0 & 0.0 & 0.0 & 0.0 & 0.0 & 0.0 & 0.0 & 0.0 & & \\
\hline Delta & 0 & 0.0 & 0 & 0.0 & 0 & 0.0 & 0 & 0.0 & 0 & 0.0 & & \\
\hline \multicolumn{13}{|l|}{ Group II } \\
\hline Alpha & 30 & 100.0 & 27 & 90.0 & 25 & 83.3 & 24 & 80.0 & 22 & 73.3 & \multirow{4}{*}{11.78} & \multirow{4}{*}{0.16} \\
\hline Bravo & 0 & 0.0 & 3 & 10.0 & 5 & 16.7 & 3 & 10.0 & 5 & 16.7 & & \\
\hline Charlie & 0 & 0.0 & 0 & 0.0 & 0.0 & 0.0 & 3 & 10.0 & 3 & 10.0 & & \\
\hline Delta & 0 & 0.0 & 0 & 0.0 & 0 & 0.0 & 0 & 0.0 & 0 & 0.0 & & \\
\hline Fisher's exact & \multicolumn{2}{|c|}{-} & \multicolumn{2}{|c|}{0.00} & \multicolumn{2}{|c|}{0.48} & \multicolumn{2}{|c|}{0.22} & \multicolumn{2}{|c|}{0.22} & & \\
\hline P-value & \multicolumn{2}{|c|}{ - } & \multicolumn{2}{|c|}{1.00} & \multicolumn{2}{|c|}{0.79} & \multicolumn{2}{|c|}{0.90} & \multicolumn{2}{|c|}{0.89} & & \\
\hline
\end{tabular}


There was no significant difference between the two tested groups regarding the retention of restoration. In addition, the difference among the four recall periods was not significant as shown in table (8).

TABLE (8): Results of clinical evaluation regarding retention of restorations

\begin{tabular}{|c|c|c|c|c|c|c|c|c|c|c|c|c|}
\hline \multirow[t]{2}{*}{$\begin{array}{r}\text { Follow up } \\
\text { period }\end{array}$} & \multicolumn{2}{|c|}{$\begin{array}{l}\text { Baseline } \\
\text { No. (30) }\end{array}$} & \multicolumn{2}{|c|}{$\begin{array}{l}6 \text { months } \\
\text { No. (30) }\end{array}$} & \multicolumn{2}{|c|}{$\begin{array}{l}12 \text { months } \\
\text { No. (30) }\end{array}$} & \multicolumn{2}{|c|}{$\begin{array}{l}18 \text { months } \\
\text { No. (30) }\end{array}$} & \multicolumn{2}{|c|}{$\begin{array}{c}24 \text { months } \\
\text { No. (30) }\end{array}$} & \multirow{2}{*}{$\begin{array}{c}\text { Friedman } \\
\text { test }\end{array}$} & \multirow[t]{2}{*}{ P-value } \\
\hline & No & $\%$ & No & $\%$ & No & $\%$ & No & $\%$ & No & $\%$ & & \\
\hline \multicolumn{13}{|l|}{ Group I } \\
\hline Alpha & 29 & 96.7 & 28 & 93.3 & 28 & 93.3 & 25 & 83.3 & 25 & 83.3 & \multirow{4}{*}{8.38} & \multirow{4}{*}{0.40} \\
\hline Bravo & 1 & 3.3 & 2 & 6.7 & 2 & 6.7 & 5 & 16.7 & 4 & 13.3 & & \\
\hline Charlie & 0 & 0.0 & 0 & 0.0 & 0 & 0.0 & 0 & 0.0 & 1 & 3.3 & & \\
\hline Delta & 0 & 0.0 & 0 & 0.0 & 0 & 0.0 & 0 & 0.0 & 0 & 0.0 & & \\
\hline \multicolumn{13}{|l|}{ Group II } \\
\hline Alpha & 30 & 100.0 & 28 & 93.0 & 26 & 86.7 & 24 & 80.0 & 22 & 73.3 & \multirow{4}{*}{11.76} & \multirow{4}{*}{0.35} \\
\hline Bravo & 0 & 0.0 & 2 & 6.7 & 4 & 10.0 & 3 & 10.0 & 4 & 13.3 & & \\
\hline Charlie & 0 & 0.0 & 0 & 0.0 & 0 & 0.0 & 3 & 10.0 & 4 & 13.3 & & \\
\hline Delta & 0 & 0.0 & 0 & 0.0 & 0 & 0.0 & 0 & 0.0 & 0 & 0.0 & & \\
\hline Fisher's exact & \multicolumn{2}{|c|}{0.00} & \multicolumn{2}{|c|}{1.02} & \multicolumn{2}{|c|}{1.27} & \multicolumn{2}{|c|}{3.52} & \multicolumn{2}{|c|}{2.19} & & \\
\hline P-value & \multicolumn{2}{|c|}{1.00} & \multicolumn{2}{|c|}{0.60} & \multicolumn{2}{|c|}{0.53} & \multicolumn{2}{|c|}{0.17} & \multicolumn{2}{|c|}{0.53} & & \\
\hline
\end{tabular}

As shown in table (9), regarding recurrent caries and postoperative sensitivity, there was no effect of the tested time on the two criterions in both tested materials. All recall periods in both groups $100 \%$ Alpha rating was recorded, so the difference between the two groups was not computed.

TABLE (9): Results of clinical evaluation regarding recurrent caries and postoperative sensitivity.

\begin{tabular}{|c|c|c|c|c|c|c|c|c|c|c|c|c|}
\hline \multirow[t]{2}{*}{$\begin{array}{r}\text { Follow up } \\
\text { period }\end{array}$} & \multicolumn{2}{|c|}{$\begin{array}{l}\text { Baseline } \\
\text { No. (30) }\end{array}$} & \multicolumn{2}{|c|}{$\begin{array}{l}6 \text { months } \\
\text { No. }(30)\end{array}$} & \multicolumn{2}{|c|}{$\begin{array}{c}12 \\
\text { months } \\
\text { No. (30) }\end{array}$} & \multicolumn{2}{|c|}{$\begin{array}{l}18 \text { months } \\
\text { No. (30) }\end{array}$} & \multicolumn{2}{|c|}{$\begin{array}{c}24 \\
\text { months } \\
\text { No. (30) }\end{array}$} & \multirow[t]{2}{*}{$\begin{array}{c}\text { Friedman } \\
\text { test }\end{array}$} & \multirow[t]{2}{*}{ P-value } \\
\hline & No & $\%$ & No & $\%$ & No & $\%$ & No & $\%$ & No & $\%$ & & \\
\hline $\begin{array}{l}\text { Group I } \\
\text { Alpha }\end{array}$ & 30 & 100.0 & 30 & 100.0 & 30 & 100.0 & 30 & 100.0 & 30 & 100.0 & \multirow{2}{*}{ - } & \multirow[b]{2}{*}{-} \\
\hline Bravo & 0 & 0.0 & 0 & 0.0 & 0 & 0.0 & 0 & 0.0 & 0 & 0.0 & & \\
\hline $\begin{array}{c}\text { Group II } \\
\text { Alpha }\end{array}$ & 30 & 100.0 & 3 & 100.0 & 30 & 100.0 & 30 & 100.0 & 30 & 100.0 & & \\
\hline Bravo & 0 & 0.0 & 0 & 00.0 & 0 & 00.0 & 0 & 0.00 & 0 & 0.00 & & \\
\hline Fisher's exact & \multicolumn{2}{|c|}{-} & \multicolumn{2}{|c|}{-} & \multicolumn{2}{|c|}{-} & \multicolumn{2}{|c|}{-} & & & \\
\hline P-value & \multicolumn{2}{|c|}{ - } & \multicolumn{2}{|c|}{ - } & & & \multicolumn{2}{|c|}{ - } & & \\
\hline
\end{tabular}




\section{DISCUSSION}

Resin based composites are used successfully for many decades, but polymerization shrinkage and its stress is considered one of the major disadvantages of composites ${ }^{[4,20]}$. Restoration placement techniques are broadly documented as a main issue in the reduction of the shrinkage stresses ${ }^{[8,10]}$. Incremental filling techniques of composite resin are generally favoured to obtain effective marginal seal and decrease the clinical consequences of polymerization shrinkage and stress ${ }^{[23]}$. Incremental techniques have been suggested to compensate for the polymerization shrinkage of composites. The bulk-fill resin based composites have been used in dentistry in the last few years ${ }^{[24]}$. They allow placement of composite up to 4 or $5 \mathrm{~mm}$ thick increments and curing in one step, the procedure is less time-consuming than incremental layering technique ${ }^{[25]}$. There are many invitro studies that evaluated the bulk fill composite resin and compared it to incremental fill composite regarding many aspects ${ }^{[10,25,26]}$. However, one could argue that although in vitro studies give information on different properties of the tested materials used, no in vitro studies can entirely subject the tested materials to in vivo situation and different oral challenges. Thus, the correlation between the results of in vitro studies and clinical studies has to be questioned and the results of in vitro studies should be supported by clinical research ${ }^{[27]}$. In the present in vivo study, all the tested criteria were evaluated after placement of restorations, at six months, at twelve months, at eighteen months and finally at two years. The modified USPHS criteria, an established method used in clinical investigation, was used in the current study to evaluate the restorations ${ }^{[28]}$. The modified USPHS criteria are considered the most commonly used system for evaluating the most important criteria of direct restorations. In the current study, acceptable results of marginal integrity were recorded during the first 18 months. The results were consistent with previous in vivo and in vitro studies, where they found that there was no significant difference regarding marginal integrity when bulk fill composite was compared with the conventional incremental fill ${ }^{[29-31]}$. Even after examining the epoxy resin replicas using electron microscope, the bulk fill composite exhibited adequate marginal integrity and behaved similarly to conventional composite ${ }^{[2]}$. Despite it was shown that at the end of follow up period Charlie score was recorded in $3.3 \%$ of the restorations restored with Tetric Evo Ceram bulk fill in group I and in $6.7 \%$ of group II, the restorations of the bulk fill composite exhibited superior scores throughout the study. There was no significant difference between Tetric $\mathrm{N}$-Ceram and Tetric Evo Ceram bulk fill regarding marginal integrity. The findings of the current study came in coincidence with the study carried out by van Dijken and Pallesen and the study carried out by Akah et al., Bayraktar et $\mathrm{al}^{[32,33]}$. demonstrated that all tested materials under examination in their study (Clearfil Photo Posterior, Filtek Bulk-Fill Flowable and Filtek P60, Tetric EvoCeram BulkFill, and SonicFill) showed satisfactory marginal integrity after twelve months clinical follow up. It has been reported in many studies that there was intimate relationship between integrity of restorations margins and polymerization contraction and polymerization shrinkage stress ${ }^{[34-36]}$. Marginal integrity of Tetric EvoCeram bulk fill restorations may be related to patented shrinkage stress. Relievers are integrated into the filler composition and give the least combination of polymerization shrinkage and stress when compared to competitive incremental fill materials. This allowed Tetric EvoCeram Bulk Fill to achieve better marginal adaptation and the results were comparable to the incremental fill composite. Incremental application of composite resin is considered one of the methods that decrease polymerization contraction stress (technical data) ${ }^{[37,38]}$.

One of the reasons responsible for marginal integrity and marginal discoloration is the adhesive type. The adhesive used in this study was the same type (self etch adhesive ) which might be an 
explanation for the results of current study regarding marginal integrity ${ }^{[37,38]}$.

On the other hand, the results of the current study did not correlate with a recent study conducted by Yazici et al., ${ }^{[14]}$. They found improved clinical performance of bulk fill composite in terms of marginal adaptation where there was a significant difference between bulk fill (Tetric EvoCeram Bulk Fill) and conventional incremental fill resin composite (Filtek Ultimate) at the end of 36 months. This might be due to the difference regarding the duration of evaluation period where in the current study the evaluation ended at 24 months. Marginal discoloration is generally related to the defects of marginal integrity. So, the marginal discoloration recorded in the same restoration showed loss of marginal integrity. This finding in the current study was supported by many other studies ${ }^{[30,39]}$.

Regarding the surface roughness in the present study, Alpha score of group I was 100.0\%, $93.3 \%$ and $93.3 \%, 83.3 \%$ and $83.3 \%$ at baseline, six months, 12 months, 18 months and 24 months respectively. In group $\Pi$ it was $100.0 \%, 90 \%$ and $83.3 \%, 80 \%$ and $80 \%$ respectively. Corresponding the same recall periods, the percentage of rough surfaces were more in group $\Pi$. This may be attributed to incorporation of voids during incremental restoration. At the end of 24 months, the difference was not significant between the two tested groups and the results of our study came in coincidence with many other studies $^{[13,15]}$. Surface roughness is a very important criterion where it is known that increased surface roughness leads to surface staining and color change as well as increased plaque accumulation and attachment of bacteria to the restoration ${ }^{[40]}$. The size of filler particle is one of the significant factors that determine the smoothness of restorations. Tetric $\mathrm{N}-\mathrm{Ceram}$ and Tetric EvoCeram Bulk Fill include features of nanotechnology. "Nano additives" and nano filler have been included in a targeted fashion. The rheological modifier contained in Tetric $\mathrm{N}$-Ceram is an example of such a nano additive. As in Tetric Ceram, this modifier is responsible for the material's viscosity and good pliability and attain smooth surface ${ }^{[41]}$ (Technical data). The results of surface roughness in the current study were comparable with that of color matching since increased surface roughness leads to change in color of the restoration. Almost the same restorations showed a change for both criterions, and the results agreed with previous studies ${ }^{[42]}$. In the current study, the differences were not significant between the two tested materials regarding both criterions. The results were similar to other studies carried out by Yazici et al., and Bayraktar et al., ${ }^{[13,48]}$.

In the present study, it was found that there was no significant difference among the groups at each recall period regarding loss of anatomic form. The explanation of this finding may be related to the existence of methacrylate based resin matrix in both tested materials which is the main component responsible for wear and subsequent loss of anatomic form. In addition, the same intensity of light curing was used in the current study. This was supported by in vitro studies conducted by Alkhudhairy and Zhang et al. ${ }^{[43,44]}$.

At the end of the 18 months period in this study, $10 \%$ of restorations in group II exhibited Charlie score, whereas in group I only one restoration scored Charlie. The difference between the two groups was not significant. There was no statistically significant difference along the tested periods in both groups. The results were in concurrence with a research carried out by Bayraktar et al. ${ }^{[15]}$. In their study, they explained the loss of retention of restorations as a result of a technical fault when placing the restorative material. This was compared with baseline scores and was not deemed to be of statistical significance. On the other hand, the results of our study were not comparable with another study ${ }^{[13]}$, where the retention rate was $100 \%$. The Mismatch of results might be due to the dissimilarity in the design of cavity preparation whereas the cavity preparation in the other study was a slot preparation. 
Over the course of this study, none of the participating cases complained of hypersensitivity or exhibited recurrence of caries. Other than some expectations that curing of composite in bulk may lead to incomplete curing and postoperative sensitivity, this was not observed. This can be interpreted by the patented polymerization booster which enabled Tetric EvoCeram bulk fill to be a material that can be placed in one increment while maintaining natural esthetics. Tetric EvoCeram bulk fill doesn't rely on an elevated level of initiators or on a highly translucent material for $4 \mathrm{~mm}$ depth of cure, so complete curing was achieved without any compromising of the handling and esthetics ${ }^{[45]}$. The bulk fill resin Tetric EvoCeram bulk fill composite resin used in the current study depends on a recent photoinitiator system, Ivocerin, a dibenzoyl germanium compound that is characterized by superior photocuring activity than camphorquinone. This new photoinitiator system absorbs visible light over a wider range of wavelengths from 370 to $460 \mathrm{~nm}{ }^{[3,46]}$. Absence of postoperative sensitivity may be attributed to self etch adhesive used with all restorations ${ }^{[28]}$. In addition, the application of liner in deep cavities participated in absence of postoperative sensitivity ${ }^{[47],[48]}$. The results of current study came in coincidence with other studies where nearly all restorations showed excellent performance in terms of postoperative sensitivity ${ }^{[13,48]}$.

Absence of recurrent caries in both groups might be related to the complete removal of caries during preparation, and the patients selected to participate in this study presented with good oral hygiene.

On the other hand, previous studies ${ }^{[49]}$ showed total of five secondary caries recorded. This could be related to marginal adaptation problems recorded in their study and local faults, such as saliva contamination, when placing the restoration. For our study, use of rubber dam for isolation may be the reason that prevented this from happening.

Despite that two-year clinical evaluation may provide valuable information on the clinical performance of restorative materials and their catastrophic failure ${ }^{[50]}$, long-term evaluation may be necessary for more accurate results.

\section{CONCLUSION}

Within the limitations of this study Bulk fill composite (Tetric EvoCeram bulk fill) and incremental fill composite resin (Tetric N-Ceram Nano-hybrid) exhibited comparable acceptable clinical performance after two years of evaluation.

\section{REFERENCES}

1. Bucuta, S. and N. Ilie, Light transmittance and micromechanical properties of bulk fill vs. conventional resin based composites. Clinical oral investigations, 2014. 18(8): p. 1991-2000.

2. Campos, E. , Ardu, S., Lefever, D., Jassé, F. F., Bortolotto, T., \& Krejci, I., Marginal adaptation of class II cavities restored with bulk-fill composites. Journal of dentistry, 2014. 42(5): p. 575-581.

3. Moszner, N., Fischer, U. K., Ganster, B., Liska, R., \& Rheinberger, V., Benzoyl germanium derivatives as novel visible light photoinitiators for dental materials. Dental Materials, 2008. 24(7): p. 901-907.

4. Kleverlaan, C.J. and A.J. Feilzer, Polymerization shrinkage and contraction stress of dental resin composites. Dental Materials, 2005. 21(12): p. 1150-1157.

5. Fleming, G. J. P., Hall, D. P., Shortall, A. C. C., \& Burke, F. J. T., Cuspal movement and microleakage in premolar teeth restored with posterior filling materials of varying reported volumetric shrinkage values. Journal of Dentistry, 2005. 33(2): p. 139-146.

6. Park, J., Chang, J., Ferracane, J., \& Lee, I. B., How should composite be layered to reduce shrinkage stress: incremental or bulk filling? Dental Materials, 2008. 24(11): p. 1501-1505.

7. Kwon, Y., J. Ferracane, and I.-B. Lee, Effect of layering methods, composite type, and flowable liner on the polymerization shrinkage stress of light cured composites. Dental Materials, 2012. 28(7): p. 801-809.

8. Bicalho, A. A., Valdívia, A. D. C. M., Barreto, B. D. C., Incremental filling technique and composite materialPart I: Cuspal deformation, bond strength, and physical properties. Operative dentistry, 2014. 39(2): p. e71-e82. 
9. Chesterman, J., Jowett, A., Gallacher, A., \& Nixon, P., Bulk-fill resin-based composite restorative materials: a review. British dental journal, 2017. 222(5): p. 337.

10. Ilie, N., S. Bucuta, and M. Draenert, Bulk-fill resin-based composites: an in vitro assessment of their mechanical performance. Operative Dentistry, 2013. 38(6): p. 618625 .

11. Al-Harbi, F., Kaisarly, D., Bader, D., \& El Gezawi, M., Marginal integrity of bulk versus incremental fill class II composite restorations. Operative dentistry, 2016. 41(2): p. 146-156.

12. Goracci, C., et al., Polymerization efficiency and flexural strength of low-stress restorative composites. Dental materials, 2014. 30(6): p. 688-694.

13. Frankenberger, R., Schulz, M., Holl, S., Seitner, T., Matthias, J., \& Roggendorf, M. J., Bulk-Fill vs. Layered Resin Composite Restorations in Class II Cavities: SixMonth Results. University Medical Center Giessen and Marburg, 2012.

14. Yazici, A. R., Antonson, S. A., Kutuk, Z. B., \& Ergin, E., Thirty-Six-Month Clinical Comparison of Bulk Fill and Nanofill Composite Restorations. Operative Dentistry, 2017.

15. Celik, C., N. Arhun, and K. Yamanel, Clinical evaluation of resin-based composites in posterior restorations: 12-month results. European journal of dentistry, 2010. 4(1): p. 57.

16. Arhun, N., C. Celik, and K. Yamanel, Clinical evaluation of resin-based composites in posterior restorations: twoyear results. Operative dentistry, 2010. 35(4): p. 397-404.

17. Walter, R., et al., Three-Year Clinical Evaluation of a Silorane Composite Resin. Journal of Esthetic and Restorative Dentistry, 2014. 26(3): p. 179-190.

18. Kemaloglu, H., T. Pamir, and H. Tezel, A 3-year randomized clinical trial evaluating two different bonded posterior restorations: Amalgam versus resin composite. European journal of dentistry, 2016. 10(1): p. 16.

19. da Rosa Rodolpho, P. A., Cenci, M. S., Donassollo, T. A., Loguércio, A. D., \& Demarco, F. F., A clinical evaluation of posterior composite restorations: 17-year findings. Journal of dentistry, 2006. 34(7): p. 427-435.

20. Baracco, B., Perdigão, J., Cabrera, E., Giráldez, I., \& Ceballos, L., Clinical evaluation of a low-shrinkage composite in posterior restorations: one-year results. Operative dentistry, 2012.37(2): p. 117-129.
21. Hickel, R., et al., Recommendations for conducting controlled clinical studies of dental restorative materials. Clinical Oral Investigations, 2007. 11(1): p. 5-33.

22. Cvar, J.F. and G. Ryge*, Reprint of Criteria for the clinical evaluation of dental restorative materials. Clinical Oral Investigations, 2006. 10(1): p. 92-92.

23. Shibasaki, S., et al., Polymerization Behavior and Mechanical Properties of High-Viscosity Bulk Fill and Low Shrinkage Resin Composites. Operative dentistry, 2017. 42(6): p. E177-E187.

24. Durán Ojeda, G., Henríquez Gutiérrez, I., Tisi, J. P., \& Báez Rosales, A., A Novel Technique for Bulk-Fill ResinBased Restorations: Achieving Function and Esthetics in Posterior Teeth. Case reports in dentistry, 2017. 2017.

25. Oskoee, S. S., Bahari, M., Navimipour, E. J., Ajami, A. A., Ghiasvand, N., \& Oskoee, A. S., Factors affecting marginal integrity of class II bulk-fill composite resin restorations. Journal of dental research, dental clinics, dental prospects, 2017. 11(2): p. 101.

26. Mosharrafian, S., A. Heidari, and P. Rahbar, Microleakage of Two Bulk Fill and One Conventional Composite in Class II Restorations of Primary Posterior Teeth. Journal of dentistry (Tehran, Iran), 2017. 14(3): p. 123.

27. Gemalmaz, D. and D. Kükrer, In vivo and in vitro evaluation of marginal fit of class II ceromer inlays. Journal of oral rehabilitation, 2006. 33(6): p. 436-442.

28. PerdigãO, J., S. Geraldeli, and J. Hodges, Total-etch versus self-etch adhesive: Effect on postoperative sensitivity. Journal of Esthetic and Restorative Dentistry, 2013. 25(4): p. 284-285.

29. Benetti, A. R., Havndrup-Pedersen, C., Honoré, D., Pedersen, M. K., \& Pallesen, U., Bulk-fill resin composites: polymerization contraction, depth of cure, and gap formation. Operative dentistry, 2015. 40(2): p. 190-200.

30. Alkurdi, R.M. and S.A. Abboud, Clinical evaluation of class II composite: Resin restorations placed by two different bulk-fill techniques. Journal of Orofacial Sciences, 2016.8(1): p. 34 .

31. Scotti, N., et al., Microleakage at enamel and dentin margins with a bulk fills flowable resin. European journal of dentistry, 2014. 8(1): p. 1.

32. van Dijken, J.W. and U. Pallesen, Randomized 3-year clinical evaluation of Class I and II posterior resin restorations placed with a bulk-fill resin composite and a one-step selfetching adhesive. J Adhes Dent, 2015. 17(1): p. 81-8. 
33. Akah, M.M., L.E. Daifalla, and M.M. Yousry, Bonding of Bulk Fill versus Contemporary Resin Composites: A Systematic Review and Meta-analysis. Indian Journal of Science and Technology, 2016. 9(20).

34. Ferracane, J.L. and J.C. Mitchem, Relationship between composite contraction stress and leakage in Class V cavities. American journal of dentistry, 2003. 16(4): p. 239-243.

35. Calheiros, F. C., Sadek, F. T., Boaro, L. C. C., \& Braga, R. R., Polymerization stress related to radiant exposure and its effect on microleakage of composite restorations. Journal of dentistry, 2007. 35(12): p. 946-952.

36. Calheiros, F. C., Sadek, F. T., Braga, R. R., \& Cardoso, P. E. C., Polymerization contraction stress of low-shrinkage composites and its correlation with microleakage in class $\mathrm{V}$ restorations. Journal of dentistry, 2004. 32(5): p. 407-412.

37. Gregor, L., Dorien, L., Bortolotto, T., Feilzer, A. J., \& Krejci, I., Marginal integrity of low-shrinking versus methacrylate-based composite: effect of different one-step self-etch adhesives. Odontology, 2017. 105(3): p. 291-299.

38. Manchorova-Veleva, N.A., S.B. Vladimirov, and D.A. Keskinova, Clinical Effect of Dental Adhesive on Marginal Integrity in Class I And Class II Resin-Composite Restorations. Folia medica, 2015. 57(3-4): p. 250-256.

39. de Oliveira, F., et al., Two-Year Clinical Evaluation of a Nanofilled Etch-and-Rinse and a Self-Etch Adhesive System Containing MDPB and Fluoride in Non-carious Cervical Lesions. Compendium of continuing education in dentistry (Jamesburg, NJ: 1995), 2017. 38(3): p. e1.

40. Park, J. W., Song, C. W., Jung, J. H., Ahn, S. J., \& Ferracane, J. L., The effects of surface roughness of composite resin on biofilm formation of Streptococcus mutans in the presence of saliva. Operative dentistry, 2012. 37(5): p. 532-539.

41. Marghalani, H.Y., Effect of filler particles on surface roughness of experimental composite series. Journal of Applied Oral Science, 2010. 18(1): p. 59-67.
42. Lai, G., Zhao, L., Wang, J., \& Kunzelmann, K. H., Surface properties and color stability of incrementally-filled and bulk-fill composites after in vitro toothbrushing. American journal of dentistry, 2017.30(5): p. 262-266.

43. Alkhudhairy, F., Wear Resistance of Bulk-fill Composite Resin Restorative Materials Polymerized under different Curing Intensities. The journal of contemporary dental practice, 2017. 18(1): p. 39-43.

44. Zhang, H., Zhang, M. L., Qiu, L. H., Yu, J. T., \& Zhan, F. L, Comparison of wear resistance and flexural strength of three kinds of bulk-fill composite resins. Shanghai kou qiang yi xue $=$ Shanghai journal of stomatology, 2016. 25(3): p. 292-295.

45. Fronza, B.M., et al., Monomer conversion, microhardness, internal marginal adaptation, and shrinkage stress of bulkfill resin composites. Dental Materials, 2015. 31(12): p. 1542-1551.

46. Vivadent, I., Scientific documentation tetric evo ceram Bulk Fill. Amherst: Ivoclar Vivadent, 2013.

47. Corralo, D. and M. Maltz, Clinical and ultrastructural effects of different liners/restorative materials on deep carious dentin: a randomized clinical trial. Caries research, 2013. 47(3): p. 243-250.

48. Bayraktar, Y., Ercan, E., Hamidi, M. M., \& Çolak, H., One-year clinical evaluation of different types of bulk-fill composites. Journal of investigative and clinical dentistry, 2017. 8(2)

49. Sadeghi, M., C.D. Lynch, and N. Shahamat, Eighteenmonth clinical evaluation of microhybrid, packable and nanofilled resin composites in Class I restorations. Journal of oral rehabilitation, 2010. 37(7): p. 532-537.

50. Hoseinifar, R., et al., One Year Clinical Evaluation of a Low Shrinkage Composite Compared with a Packable Composite Resin: A Randomized Clinical Trial. Journal of dentistry (Tehran, Iran), 2017. 14(2): p. 84. 